\title{
Altered default mode, fronto-parietal and salience networks in adolescents with Internet addiction
}

Lubin Wang ${ }^{1,2}$, Hui Shen ${ }^{2}$, Yu Lei ${ }^{1}$, Ling-Li Zeng ${ }^{2}$, Fenglin Cao ${ }^{3}$, Linyan $\mathrm{Su}^{3}$, Zheng Yang ${ }^{1, *}$, Shuqiao $\mathrm{Yao}^{3, *}$, Dewen $\mathrm{Hu}^{2}{ }^{2} *$

${ }^{1}$ Cognitive and Mental Health Research Center, Beijing Institute of Basic Medical Sciences, Beijing 100850, China

${ }^{2}$ College of Mechatronics and Automation, National University of Defense Technology, Changsha, Hunan 410073, China

${ }^{3}$ The Medical Psychological Institute of the Second Xiangya Hospital, Central South University, Changsha, Hunan 410011, China

\section{*Correspondence to:}

Zheng Yang, Cognitive and Mental Health Research Center, Beijing Institute of Basic Medical Sciences, 27 Taiping Road, Beijing 100850, China

E-mail: yangzhengchina@aliyun.com

Shuqiao Yao, The Medical Psychological Institute of the Second Xiangya Hospital, Central South University, 139 Renmin (M) Road, Changsha, Hunan 410011, China E-mail: Shuqiaoyao@163.com

Dewen Hu, College of Mechatronics and Automation, National University of Defense Technology, Changsha, Hunan 410073, China

E-mail: dwhu@nudt.edu.cn 


\begin{abstract}
Internet addiction (IA) is a condition characterized by loss of control over Internet use, leading to a variety of negative psychosocial consequences. Recent neuroimaging studies have begun to identify IA-related changes in specific brain regions and connections. However, whether and how the interactions within and between the large-scale brain networks are disrupted in individuals with IA remain largely unexplored. Using group independent component analysis, we extracted five intrinsic connectivity networks (ICNs) from the resting-state fMRI data of 26 adolescents with IA and 43 controls, including the anterior and posterior default mode network (DMN), left and right fronto-parietal network (FPN), and salience network (SN). We then examined the possible group differences in the functional connectivity within each ICN and between the ICNs. We found that, compared with controls, IA subjects showed: (1) reduced inter-hemispheric functional connectivity of the right FPN, whereas increased intra-hemispheric functional connectivity of the left FPN; (2) reduced functional connectivity in the dorsal medial prefrontal cortex (mPFC) of the anterior DMN; (3) reduced functional connectivity between the SN and anterior DMN. Our findings suggest that IA is associated with imbalanced interactions among the DMN, FPN and SN, which may serve as system-level neural underpinnings for the uncontrollable Internet-using behaviors.
\end{abstract}

Keywords: Internet addiction; Functional connectivity; Default mode network; Fronto-parietal network; Salience network 


\section{Introduction}

Internet addiction (IA), also described as problematic or pathological Internet use, is characterized by loss of control regarding Internet use, and shares many aspects of substance addiction such as preoccupation, mood modification, tolerance, withdrawal, distress and functional impairments (Aboujaoude, 2010; Spada, 2014; Young, 1998). With the increasing number of Internet users, IA is becoming an important mental health issue worldwide (Cao \& Su, 2007; Niemz, Griffiths, \& Banyard, 2005). Addictive Internet use could lead to a series of negative consequences such as academic performance, social interaction, occupational interest, and behavioral problems. However, the patho-physiological and cognitive mechanisms responsible for IA remain largely unknown.

To understand the neural basis that underlies IA, a number of neuroimaging studies have been performed to investigate structural and functional brain changes associated with this non-substance-related behavioral addiction (Brand, Young, \& Laier, 2014; Zhu, Zhang, \& Tian, 2015). These studies showed that Internet-addicted individuals had lower gray matter density (Zhou, et al., 2011), abnormal white matter fractional anisotropy (Lin, et al., 2012; Yuan, et al., 2011), and impaired brain activity during performance of certain tasks (Ding, et al., 2014; Dong, Lin, Zhou, \& Lu, 2014). While the above neuroimaging methodologies have been essential for delineating brain abnormalities in specific brain regions, additional and important insight will most likely be provided by considering alterations in functional brain networks and their interactions (Volkow, Wang, Fowler, \& Tomasi, 2012).

Resting-state functional MRI (rs-fMRI) is a powerful tool for mapping large-scale brain network function and dysfunction. This method is based on the discovery that spatially distinct but functionally related brain regions have highly correlated spontaneous low-frequency $(<0.1 \mathrm{~Hz})$ blood oxygen level-dependent fluctuations, 
namely the intrinsic connectivity networks (ICNs) of the human brain (Biswal, Yetkin, Haughton, \& Hyde, 1995; Fox \& Raichle, 2007). These networks recapitulate the architecture of the known functional systems related to sensory, motor, attention, memory and other brain functions, and have been proved to play a role in human behaviors (van den Heuvel \& Hulshoff Pol, 2010). A growing number of studies have used rs-fMRI to examine neural circuitry dysfunction underlying various neurological and psychiatric disorders (Fox \& Greicius, 2010). Moreover, recent studies have also reported dysfunctions of ICNs in individuals with IA (Dong, Lin, \& Potenza, 2015; Wang, et al., 2016; Zhang, et al., 2016), suggesting that IA may be associated with system-level alterations between brain regions.

Among the ICNs, three higher-order networks have received particular attention: a fronto-parietal network (FPN) implicated in a wide range of cognitively demanding tasks; a default mode network (DMN) involved in stimulus-independent thought processes; and a salience network (SN) identifying the most subjectively relevant stimuli and allocating attention (Buckner, Andrews-Hanna, \& Schacter, 2008; Seeley, et al., 2007). Previous studies have suggested a negative correlation between FPN and DMN activities (Fox, et al., 2005), and a role of the SN in mediating activity between the DMN and FPN (Sridharan, Levitin, \& Menon, 2008). Menon (2011) further proposed a triple network model which focused on the SN, DMN, and ECN, indicating that dysfunction in one network can impact the other two networks. Recently, the triple network model has been adapted for neurobiological explanation of cognitive/affective alterations in addictive disorders (Sutherland, McHugh, Pariyadath, \& Stein, 2012). However, whether and how the interactions within and between these ICNs are disrupted in Internet-addicted individuals remain largely unexplored.

In this study, we investigated resting-state functional connectivity within and between the DMN, FPN and SN, in adolescents with IA and healthy controls. Group independent component analysis (gICA), a multivariate data-driven method, was used 
to decompose the rs-fMRI data into distinct ICNs (Calhoun, Liu, \& Adali, 2009). We assessed the IA-related changes in intrinsic brain activity by comparing spatial extension and intensity of each ICN between the two groups. Then we evaluated the IA-related changes in the temporal correlation between the time series of the ICNs, which is referred to as functional connectivity between ICNs (Jafri, Pearlson, Stevens, \& Calhoun, 2008). Based on the similarity between behavioral manifestations of IA and substance addiction (Beard \& Wolf, 2001; Young, 1998), we hypothesized individuals with IA would have some abnormal integrity of network interactions in common with other addictive disorders.

\section{Materials and methods}

\section{Participants}

The current study included 31 adolescents with IA and 50 normal controls $(\mathrm{CN})$. The IA subjects were outpatients of the Second Xiangya Hospital of Central South University while the controls were recruited from high schools in Changsha. There were no significant differences between the two groups in age $(\mathrm{CN}: 15.1 \pm 0.5$ years, IA: $15.0 \pm 1.3$ years; $p=0.52$, Wilcoxon rank sum test) and sex $(\mathrm{CN}: 35$ males/15 females, IA: 21 males/10 females; $p=0.83$, Pearson Chi-square test). The IA subjects satisfied the diagnosis of Internet dependence based on the modified Young's Diagnostic Questionnaire (YDQ) criteria (Beard \& Wolf, 2001). All the subjects received a structured clinical interview from two experienced psychiatrists, which was based on Diagnostic and Statistical Manual of Mental Disorders, Fourth Edition (DSM-IV). None of the participants in this study fulfilled any DSM-IV axis I disorders. More exclusion criteria included alcohol, nicotine or any other substance abuse. The experimental procedure was approved by the Ethics Committee of Second Xiangya Hospital of Central South University and was carried out in accordance with the Declaration of Helsinki. All subjects' caretakers signed an informed consent form prior to enrollment in the study. After excluding participants with excessive head movement, the final study groups consisted of 26 IA subjects and 43 controls. 
The modified YDQ criteria consisted of the following eight items: (1) Preoccupied with the Internet; (2) The need to use the Internet with increasing amounts of time to achieve satisfaction; (3) Repeatedly making unsuccessful efforts to control, cut back, or stop Internet use; (4) Feeling restless, moody, depressed, or irritable when attempting to cut down or stop Internet use; (5) Staying online longer than originally intended; (6) Jeopardized or risked the loss of a significant relationship, job, educational, or career opportunity because of the Internet; (7) Lying to others to conceal the extent of involvement with the Internet; (8) Using the Internet as a way of escaping from problems or of relieving a dysphoric mood. Participants who answered 'yes' to items 1 through 5 and at least any one of the remaining three items were classified as suffering from IA.

\section{MRI Acquisition}

Imaging was performed using A PHILIPS 3.0 T whole-body scanner. Axial rs-fMRI data of the whole brain were obtained within $7 \min 12 \mathrm{~s}$ with a $\mathrm{T} 2 *$-weighted gradient-echo echo-planar imaging sequence. During the resting-state scanning, the subjects were instructed to keep their eye closed, to relax and to stay awake (confirmed by subjects immediately after the experiment). The fMRI imaging parameters were: $\mathrm{TR}=2000 \mathrm{~ms}, \mathrm{TE}=30 \mathrm{~ms}$, flip angle $=90^{\circ}, 36$ slices, slice thickness $=4 \mathrm{~mm}$, no gap, matrix size $=64 \times 64$ and field of view $=240 \times 240 \mathrm{~mm}^{2}$. Each functional session contained 216 image volumes.

\section{Data Preprocessing}

The rs-fMRI data were preprocessed using the statistical parametric mapping software package (SPM8, http://www.fil.ion.ucl.ac.uk/spm). Prior to preprocessing, the first 10 volumes were discarded for magnetic saturation effect. The images were corrected for within-scan acquisition time differences between slices and realigned to the first volume to correct for interscan head motions. Subjects with more than $2 \mathrm{~mm}$ of translation or $2^{\circ}$ of rotation in any of the $\mathrm{x}, \mathrm{y}$, and $\mathrm{z}$ axes were removed from this 
study. Then, the images were normalized to the standard EPI template in the Montreal Neurological Institute (MNI) space and resliced to $3 \times 3 \times 3 \mathrm{~mm}^{3}$. The resulting images were spatially smoothed with a Gaussian filter of $6 \mathrm{~mm}$ full-width half-maximum kernel.

\section{Analysis of functional connectivity within ICNs}

After these preprocessing steps, gICA was conducted using the GIFT software (http://icatb.sourceforge.net/), which is a multi-subject analysis apporach for blind source signal separation and has been widely used to identify and quantify distributed patterns or spatial networks of correlated brain activity. For each subject, 30 spatially independent components were decomposed using gICA analysis. A selection of the DMN, FPN and SN was then conducted based on visual inspection of similarity between the spatial map of each component and ICNs described before ( $\mathrm{Li}$, et al., 2013; Seeley, et al., 2007; van den Heuvel \& Hulshoff Pol, 2010).

The individual subjects' spatial maps of each selected ICN were converted to Z values. The intensities of each spatial map indicated the degree to which a given voxel's time series is correlated with the distributed and coherent brain activity of that particular ICN (Calhoun, et al., 2009). Higher correlation values in a specific area correspond to stronger involvement of that area in the ICNs. When a group showed higher correlation values within a particular network, this was referred to as "increased functional connectivity”. For each selected ICN, voxel-wise one-sample t-test was first carried out across all subjects' spatial maps. The statistically thresholded t-value map was used to define brain regions that belong to the ICN. Functional connectivity differences within all regions of each ICN were then examined by using voxel-wise two-sample t-test between the IA and control groups.

The statistical map was thresholded at $p<0.005, \mathrm{~T}>2.56$ at the voxel level, then corrected for multiple comparisons to $p<0.05$ using a cluster threshold. AFNI's AlphaSim was used to compute the cluster size threshold based on 1,000 Monte Carlo 
simulations (http://afni.nimh.nih.gov/pub/dist/doc/manual/AlphaSim.pdf). Intrinsic smoothness of the data was estimated using AFNI's 3dFWHMx. For the one-sample ttests, the simulations were performed on the brain mask defined by the GIFT software, and yielded a minimum cluster size of $46-53$ voxels. For the two-sample t-tests, the simulations were performed on a mask of the ICN maps, and yielded a minimum cluster size of 28-31 voxels.

\section{Analysis of functional connectivity between ICNs}

For each individual, time series of all the voxels in each ICN were extracted and averaged. The mean time series of the selected ICNs were temporally band-pass filtered $(0.01-0.1 \mathrm{~Hz})$, followed by linear detrending to remove any residual drift. Nine nuisance signals were further removed from the time courses via linear regression, including signals averaged from white matter, cerebrospinal fluid, and the whole brain, and six parameters obtained by head motion correction. This regression procedure was performed to reduce spurious variance unlikely to reflect neuronal activity. The temporal relationship between ICN time series was evaluated by using Pearson's correlation coefficient. Fisher's z-transform was applied to the correlation values to ensure normality. For each pair of ICNs, we compared the correlation values in the IA subjects and controls, to study possible IA-related differences of functional connectivity between ICNs.

\section{Results}

\section{ICN maps}

Figure 1 shows the spatial maps of the five selected ICNs obtained from the rs-fMRI data of all the subjects by gICA. Among these ICNs, the left FPN includes a spatial pattern predominantly composed of left hemisphere dorsolateral prefrontal cortex and inferior parietal lobule, while the right FPN mainly includes corresponding areas in the contralateral hemisphere. The anterior DMN constitutes a large portion of the medial prefrontal cortex, anterior cingulate cortex, and also a small proportion of the 
posterior cingulate cortex/precuneus and angular gyrus, which comprises main areas of the posterior DMN. The SN mainly consists of regions of the dorsal anterior cingulate cortex and anterior insula.

\section{IA-related differences of functional connectivity within the ICNs}

For each RSN, we used a voxel-wise two-sample t test to examine the IA-related differences of functional connectivity within the ICNs. Brain areas exhibiting significant group differences are shown in Figure 2. Compared with the controls, the IA subjects showed increased functional connectivity in the left inferior frontal gyrus (MNI: -51, 5, 32; BA 9) and left angular gyrus (MNI: -39, -70, 26; BA 39) of the left FPN; whereas reduced functional connectivity in the left inferior parietal lobule (MNI: $-60,-46,35 ;$ BA 40) of the right FPN. Moreover, the IA subjects showed reduced functional connectivity in the dorsal medial prefrontal cortex (mPFC) (MNI: 0, 26, 56; BA 6) of the anterior DMN. Using the same significance level, no IA-related functional connectivity difference was observed in the other ICNs.

\section{IA-related differences of functional connectivity between the ICNs}

The above network assignment also provides the basis for assessing the functional connectivity patterns between ICNs. Figure 3A, B shows the inter-connectivity for all five ICNs. For both groups, the subsystems of the DMN (anterior DMN vs. posterior DMN) and FPN (left FPN vs. right FPN ) showed high positive connectivity, while the posterior DMN showed negative connectivity with the SN. When examining differences in inter-connectivity between the two groups, we observed significantly reduced functional connectivity between the $\mathrm{SN}$ and anterior $\mathrm{DMN}(\mathrm{t}=2.7, p=0.008)$ in the IA group compared with the $\mathrm{CN}$ group (Figure 3C, D). We did not find significant difference between the two groups in any of the other inter-connections regarding these ICNs (all $p$ values $>0.05$ ), which indicates that these connections are not likely to be relevant to the Internet addiction. 


\section{Discussion}

In this study, we investigated IA-related changes of functional connectivity within and between the DMN, FPN, and SN. We found that, compared with controls, IA subjects showed reduced inter-hemispheric functional connectivity of the right FPN, whereas increased intra-hemispheric functional connectivity of the left FPN. IA subjects also showed reduced functional connectivity in the anterior part of the DMN, as well as decreased interactions between the $\mathrm{SN}$ and anterior DMN. Converging evidence suggests that various mental disorders result from a potential functional reorganization of large-scale brain networks (Fox \& Greicius, 2010). Our study further showed the alteration of the DMN, FPN, and SN and their interactions in individuals with IA, which may serve as system-level neural underpinnings for the uncontrollable Internet-using behaviors.

\section{Intra-connectivity within the FPN}

Compared to controls, individuals with IA showed lower functional connectivity in the left inferior parietal lobule of the right FPN. Deficit in neural systems associated with cognitive control represents a typical feature for substance addiction. It has been demonstrated that abnormal functional connectivity between FPN regions is present in both heroin- and cocaine-addicted samples (Kelly, et al., 2011; Yuan, et al., 2010), and is associated with approaching behaviors in substance dependence (Krmpotich, et al., 2013). Previous studies also found that individuals with IA showed abnormal brain activation during cognitive tasks (Ding, et al., 2014; Dong, DeVito, Du, \& Cui, 2012), as well as decreased resting-state functional connectivity (Dong, et al., 2015) in the FPN. In combination with previous findings, our study suggests decreased coherence of brain activity between FPN regions in different hemispheres in IA subjects, which may underlie impaired executive function and weakened inhibition control of Internet-using behaviors. We also observed increased functional connectivity in the ipsolateral regions of the left FPN. It is possible that IA is associated with shared disturbances of lower interhemispheric and higher intrahemispheric functional 
connectivity. However, the mechanisms of this phenomenon should be further investigated.

\section{Intra-connectivity within the DMN}

We found that individuals with IA showed lower functional connectivity in the dorsal mPFC of the anterior DMN. Recently, IA-related alteration of the DMN has attracted considerable attention. For instance, by using the same gICA method, Wang et al. (2016) reported altered modulation in the DMN and less engagement of the FPN in IA subjects during a probability discounting task. Another study also found a significant association between mPFC functional connectivity and individual differences in Internet tendency by using seed-based correlation method (Li, et al., 2015). The result that IA subjects show relatively decreased functional connectivity in the DMN is also consistent with findings in drug addicts (Ma, et al., 2011; Zhai, et al., 2015). Therefore, the DMN abnormalities may underlie the disturbance of attentional orientation and self-referential processing for both substance and behavioral addictions (Volkow, et al., 2012).

\section{Inter-connectivity between the $\mathrm{SN}$ and $\mathrm{DMN}$}

For network interactions, we found that individuals with IA exhibited reduced functional connectivity between the SN and anterior DMN. Recently, a triple network model, anchored in the DMN, FPN and $\mathrm{SN}$, has been proposed to explain various psychiatric disorders, including addiction (Menon, 2011; Sutherland, et al., 2012). In the context of this model, the SN is involved in mediating dynamic activity between the DMN and FPN based on attribution of saliency to internal and external stimuli. The functional connectivity involving the insula, a core region in $\mathrm{SN}$, has been reported to be altered in IA subjects, and related to symptom severity (Zhang, et al., 2016). Reduced inter-connectivity between the SN and DMN suggests that individuals with IA may fail to adequately suppress DMN activity during internally oriented processing, resulting in poorly controlled preoccupations or urges regarding Internet use. Interestingly, our result is also consistent with a recent study that found 
disruptive changes of interaction between the $\mathrm{SN}$ and DMN in cocaine addiction (Liang, et al., 2015), supporting the hypothesis that IA may share similar neurobiological abnormalities with other addictive disorders.

\section{Limitations}

There were several potential limitations in this study. Firstly, we employed a relative short duration of rs-fMRI scan (about $7 \mathrm{~min}$ ) in this study, to ensure the compliance of the adolescent subjects to the experiment. The reliability of functional connectivity estimates can be improved by increasing the imaging duration (Birn, et al., 2013). Secondly, our findings should be considered specific to adolescents with IA, a stage at which the brain is still developing. Examination of functional connectivity in adults could further represent the IA-related brain changes at maturity. Thirdly, we did not quantitatively evaluate the participants' addiction severity in this study, thus could not directly explore the brain-behavior relationships. Finally, to elucidate the shared and distinct neurobiology of substance addiction and behavioral addiction such as IA, further research investigating patients from both clinical populations should be conducted.

\section{Conclusion}

In conclusion, this study found that IA was associated with altered intrinsic brain activity in the FPN and DMN, as well as reduced functional connectivity between the SN and anterior DMN. The imbalanced interactions within and between large-scale brain networks may serve as system-level neural underpinnings for the uncontrollable Internet-using behaviors. Moreover, our findings suggest that IA may share similar neurobiological abnormalities with other addictive disorders. 


\section{References}

Aboujaoude, E. (2010). Problematic Internet use: an overview. World Psychiatry, 9, 85-90.

Beard, K. W., \& Wolf, E. M. (2001). Modification in the proposed diagnostic criteria for Internet addiction. Cyberpsychol Behav, 4, 377-383.

Birn, R. M., Molloy, E. K., Patriat, R., Parker, T., Meier, T. B., Kirk, G. R., Nair, V. A., Meyerand, M. E., \& Prabhakaran, V. (2013). The effect of scan length on the reliability of resting-state fMRI connectivity estimates. Neuroimage, 83, 550558.

Biswal, B., Yetkin, F. Z., Haughton, V. M., \& Hyde, J. S. (1995). Functional connectivity in the motor cortex of resting human brain using echo-planar MRI. Magnetic Resonance in Medicine, 34, 537-541.

Brand, M., Young, K. S., \& Laier, C. (2014). Prefrontal control and internet addiction: a theoretical model and review of neuropsychological and neuroimaging findings. Front Hum Neurosci, 8, 375.

Buckner, R. L., Andrews-Hanna, J. R., \& Schacter, D. L. (2008). The brain's default network - Anatomy, function, and relevance to disease. Annals of the New York Academy of Sciences, 1124, 1-38.

Calhoun, V. D., Liu, J., \& Adali, T. (2009). A review of group ICA for fMRI data and ICA for joint inference of imaging, genetic, and ERP data. Neuroimage, 45, S163-172.

Cao, F., \& Su, L. (2007). Internet addiction among Chinese adolescents: prevalence and psychological features. Child: Care, Health and Development, 33, 275281.

Ding, W. N., Sun, J. H., Sun, Y. W., Chen, X., Zhou, Y., Zhuang, Z. G., Li, L., Zhang, Y., Xu, J. R., \& Du, Y. S. (2014). Trait impulsivity and impaired prefrontal impulse inhibition function in adolescents with internet gaming addiction revealed by a Go/No-Go fMRI study. Behavioral and Brain Functions, 10, 20.

Dong, G., DeVito, E. E., Du, X. X., \& Cui, Z. Y. (2012). Impaired inhibitory control 
in 'internet addiction disorder': A functional magnetic resonance imaging study. Psychiatry Research: Neuroimaging, 203, 153-158.

Dong, G., Lin, X., \& Potenza, M. N. (2015). Decreased functional connectivity in an executive control network is related to impaired executive function in Internet gaming disorder. Prog Neuropsychopharmacol Biol Psychiatry, 57, 76-85.

Dong, G., Lin, X., Zhou, H., \& Lu, Q. (2014). Cognitive flexibility in internet addicts: fMRI evidence from difficult-to-easy and easy-to-difficult switching situations. Addictive Behaviors, 39, 677-683.

Fox, M. D., \& Greicius, M. (2010). Clinical applications of resting state functional connectivity. Front Syst Neurosci, 4, 19.

Fox, M. D., \& Raichle, M. E. (2007). Spontaneous fluctuations in brain activity observed with functional magnetic resonance imaging. Nat Rev Neurosci, 8, 700-711.

Fox, M. D., Snyder, A. Z., Vincent, J. L., Corbetta, M., Van Essen, D. C., \& Raichle, M. E. (2005). The human brain is intrinsically organized into dynamic, anticorrelated functional networks. Proc Natl Acad Sci U S A, 102, 9673-9678.

Jafri, M. J., Pearlson, G. D., Stevens, M., \& Calhoun, V. D. (2008). A method for functional network connectivity among spatially independent resting-state components in schizophrenia. Neuroimage, 39, 1666-1681.

Kelly, C., Zuo, X.-N., Gotimer, K., Cox, C. L., Lynch, L., Brock, D., Imperati, D., Garavan, H., Rotrosen, J., Castellanos, F. X., \& Milham, M. P. (2011). Reduced interhemispheric resting state functional connectivity in cocaine addiction. Biol Psychiatry, 69, 684-692.

Krmpotich, T. D., Tregellas, J. R., Thompson, L. L., Banich, M. T., Klenk, A. M., \& Tanabe, J. L. (2013). Resting-state activity in the left executive control network is associated with behavioral approach and is increased in substance dependence. Drug and Alcohol Dependence, 129, 1-7.

Li, B., Liu, L., Friston, K. J., Shen, H., Wang, L., Zeng, L. L., \& Hu, D. (2013). A treatment-resistant default mode subnetwork in major depression. Biol Psychiatry, 74, 48-54. 
Li, W. W., Li, Y. D., Yang, W. J., Zhang, Q. L., Wei, D. T., Li, W. F., Hitchman, G., \& Qiu, J. (2015). Brain structures and functional connectivity associated with individual differences in Internet tendency in healthy young adults. Neuropsychologia, 70, 134-144.

Liang, X., He, Y., Salmeron, B. J., Gu, H., Stein, E. A., \& Yang, Y. H. (2015). Interactions between the salience and default-mode networks are disrupted in cocaine addiction. $J$ Neurosci, 35, 8081-8090.

Lin, F., Zhou, Y., Du, Y., Qin, L., Zhao, Z., Xu, J., \& Lei, H. (2012). Abnormal white matter integrity in adolescents with internet addiction disorder: a tract-based spatial statistics study. PLoS One, 7, e30253.

Ma, N., Liu, Y., Fu, X., Li, N., Wang, C., Zhang, H., Qian, R., Xu, H., Hu, X., \& Zhang, D. (2011). Abnormal brain default-mode network functional connectivity in drug addicts. PLoS One, 6, e16560.

Menon, V. (2011). Large-scale brain networks and psychopathology: a unifying triple network model. Trends Cogn Sci, 15, 483-506.

Niemz, K., Griffiths, M., \& Banyard, P. (2005). Prevalence of pathological Internet use among university students and correlations with self-esteem, the General Health Questionnaire (GHQ), and disinhibition. CyberPsychology \& Behavior, $8,562-570$.

Seeley, W. W., Menon, V., Schatzberg, A. F., Keller, J., Glover, G. H., Kenna, H., Reiss, A. L., \& Greicius, M. D. (2007). Dissociable intrinsic connectivity networks for salience processing and executive control. J Neurosci, 27, 23492356.

Spada, M. M. (2014). An overview of problematic Internet use. Addictive Behaviors, 39, 3-6.

Sridharan, D., Levitin, D. J., \& Menon, V. (2008). A critical role for the right frontoinsular cortex in switching between central-executive and default-mode networks. Proc Natl Acad Sci U S A, 105, 12569-12574.

Sutherland, M. T., McHugh, M. J., Pariyadath, V., \& Stein, E. A. (2012). Resting state functional connectivity in addiction: Lessons learned and a road ahead. 
Neuroimage, 62, 2281-2295.

van den Heuvel, M. P., \& Hulshoff Pol, H. E. (2010). Exploring the brain network: a review on resting-state fMRI functional connectivity. Eur Neuropsychopharmacol, 20, 519-534.

Volkow, N. D., Wang, G. J., Fowler, J. S., \& Tomasi, D. (2012). Addiction circuitry in the human brain. Annu Rev Pharmacol Toxicol, 52, 321-336.

Wang, L., Wu, L., Lin, X., Zhang, Y., Zhou, H., Du, X., \& Dong, G. (2016). Dysfunctional default mode network and executive control network in people with Internet gaming disorder: independent component analysis under a probability discounting task. European Psychiatry, 34, 36-42.

Young, K. S. (1998). Internet addiction: the emergence of a new clinical disorder. CyberPsychology \& Behavior, 1, 237-244.

Yuan, K., Qin, W., Dong, M., Liu, J., Sun, J., Liu, P., Zhang, Y., Wang, W., Wang, Y., Li, Q., Zhao, L., von Deneen, K. M., Liu, Y., Gold, M. S., \& Tian, J. (2010). Gray matter deficits and resting-state abnormalities in abstinent heroindependent individuals. Neuroscience Letters, 482, 101-105.

Yuan, K., Qin, W., Wang, G., Zeng, F., Zhao, L., Yang, X., Liu, P., Liu, J., Sun, J., von Deneen, K. M., Gong, Q., Liu, Y., \& Tian, J. (2011). Microstructure abnormalities in adolescents with internet addiction disorder. PLoS One, 6, e20708

Zhai, T., Shao, Y., Chen, G., Ye, E., Ma, L., Wang, L., Lei, Y., Chen, G., Li, W., Zou, F., Jin, X., Li, S., \& Yang, Z. (2015). Nature of functional links in valuation networks differentiates impulsive behaviors between abstinent heroindependent subjects and nondrug-using subjects. Neuroimage, 115, 76-84.

Zhang, J. T., Yao, Y. W., Li, C. S., Zang, Y. F., Shen, Z. J., Liu, L., Wang, L. J., Liu, B., \& Fang, X. Y. (2016). Altered resting-state functional connectivity of the insula in young adults with Internet gaming disorder. Addict Biol, 21, 743-751.

Zhou, Y., Lin, F. C., Du, Y. S., Qin, L. D., Zhao, Z. M., Xu, J. R., \& Lei, H. (2011). Gray matter abnormalities in Internet addiction: a voxel-based morphometry study. Eur J Radiol, 79, 92-95. 
Zhu, Y., Zhang, H., \& Tian, M. (2015). Molecular and functional imaging of internet addiction. Biomed Res Int, 2015, 378675. 


\section{Figure Legends}

Figure 1. Five components generated from group ICA of the resting state data were identified as the left and right fronto-parietal network (FPN), anterior and posterior default mode network (DMN), and salience network (SN). L and R indicated the left and right hemisphere of the brain.

Figure 2. Group comparisons of functional connectivity within the ICNs. (A) Sagittal, coronal, and axial views of brain regions showing altered functional connectivity within the left FPN, right FPN and anterior DMN in the IA group relative to the CN group. (B) Bar graphs plotting the mean connectivity strength of the regions in panel A. The error bars represent standard deviation. $\mathrm{CN}$, control; IA, Internet addiction; IFG, inferior frontal gyrus; IPL, inferior parietal lobule; dmPFC, dorsal medial prefrontal cortex.

Figure 3. Group comparisons of functional connectivity between the ICNs. (A) Mean connectivity strength matrix of the IA group. (B) Mean connectivity strength matrix of the $\mathrm{CN}$ group. (C) T-statistics for the significance of any differences between groups for all connections. (D) Bar plots for significant group differences in interconnectivity between the aDMN and SN. Top color bars represent connectivity strength, while bottom color bar represents T-value. The error bars represent standard deviation. $\mathrm{CN}$, control; IA, Internet addiction; aDMN, anterior DMN; pDMN, posterior DMN; IFPN, left FPN; rFPN, right FPN. *: $p<0.01$. 
Left FPN
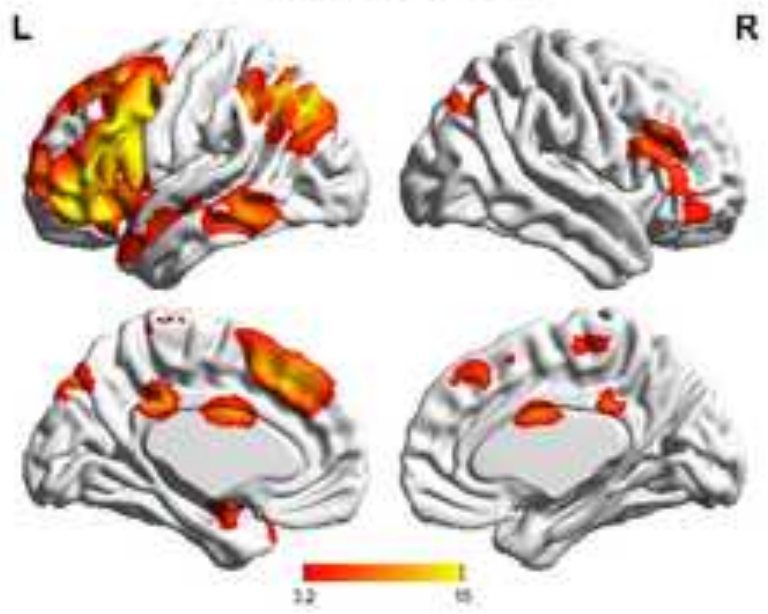

Right FPN
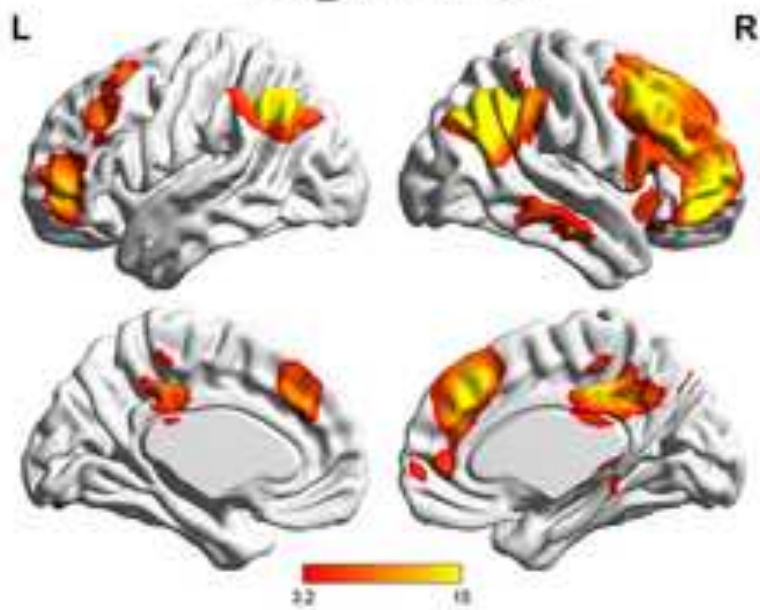

Anterior DMN
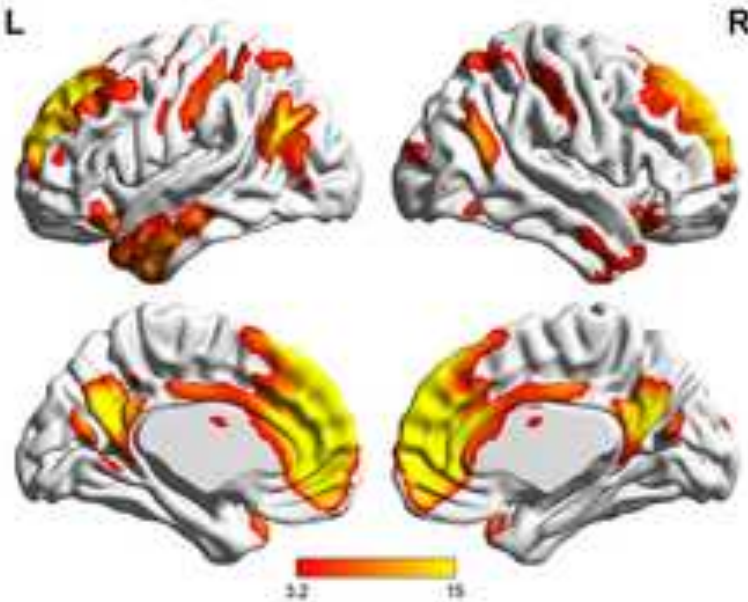

Posterior DMN
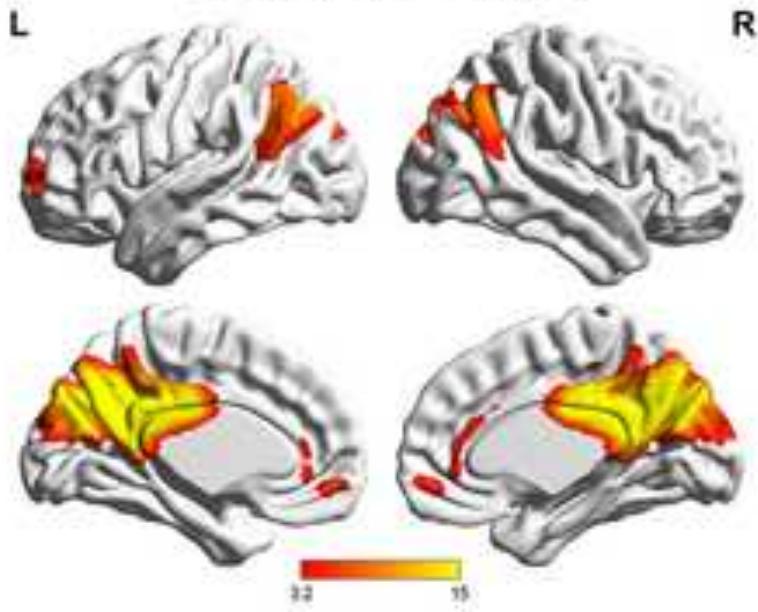

SN
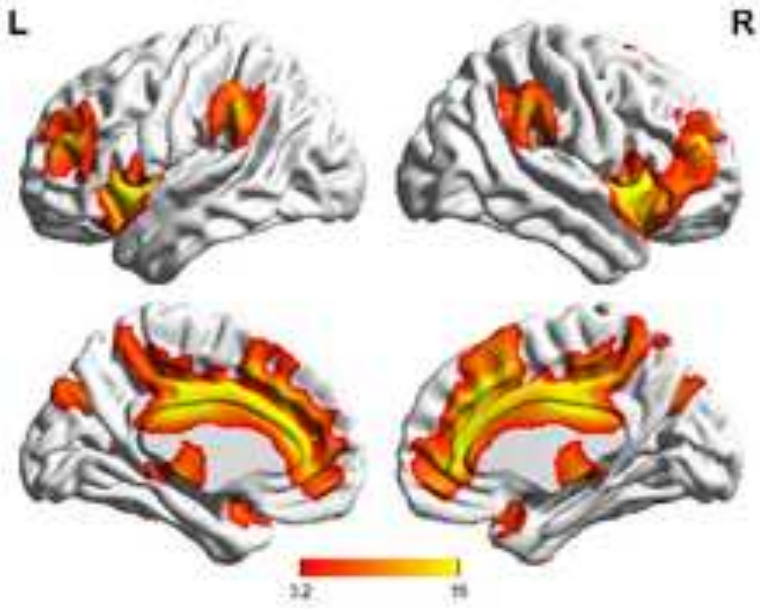
(A)

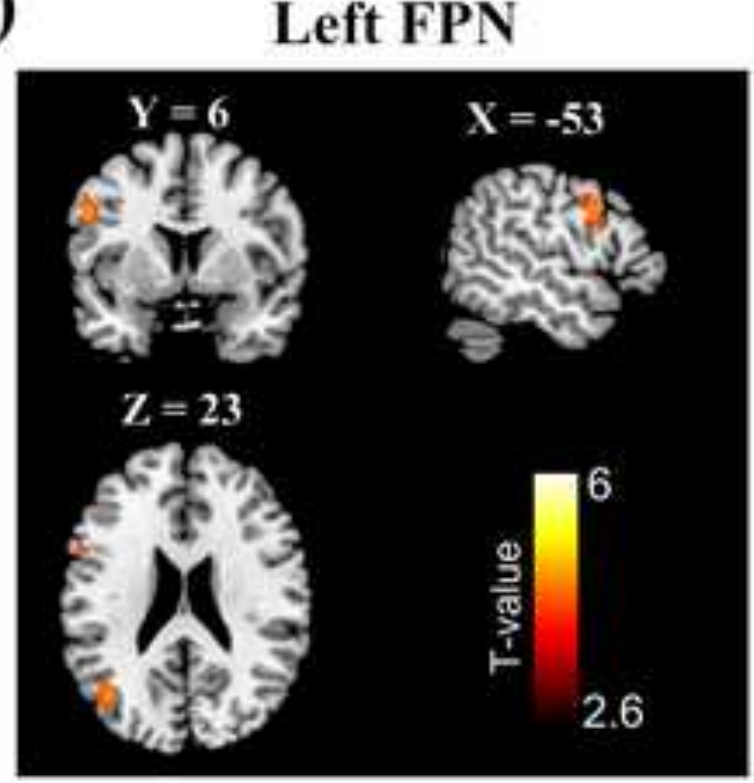

(B)

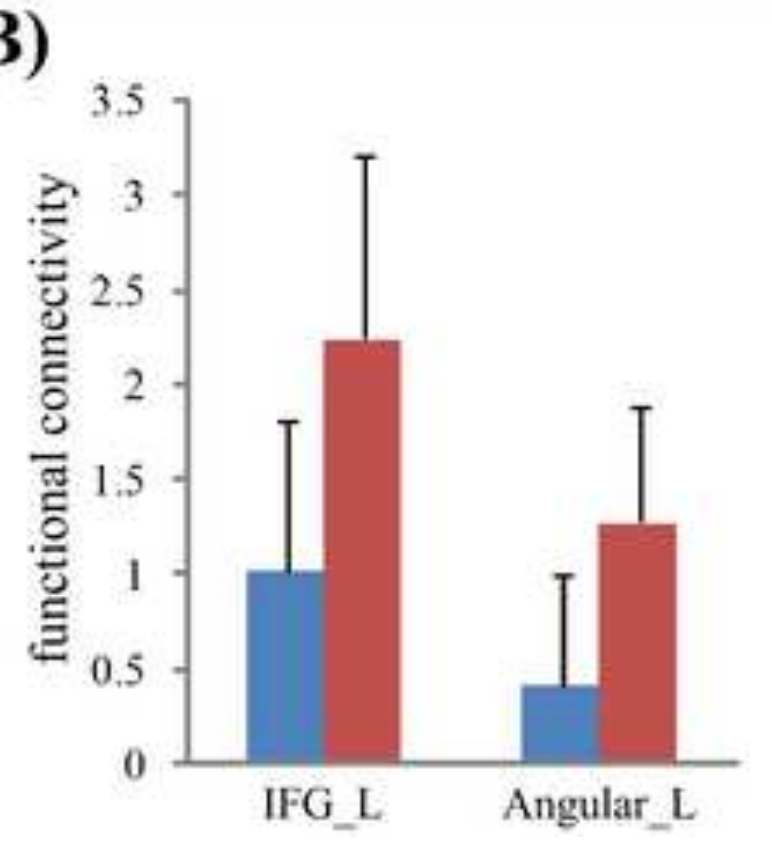

Right FPN
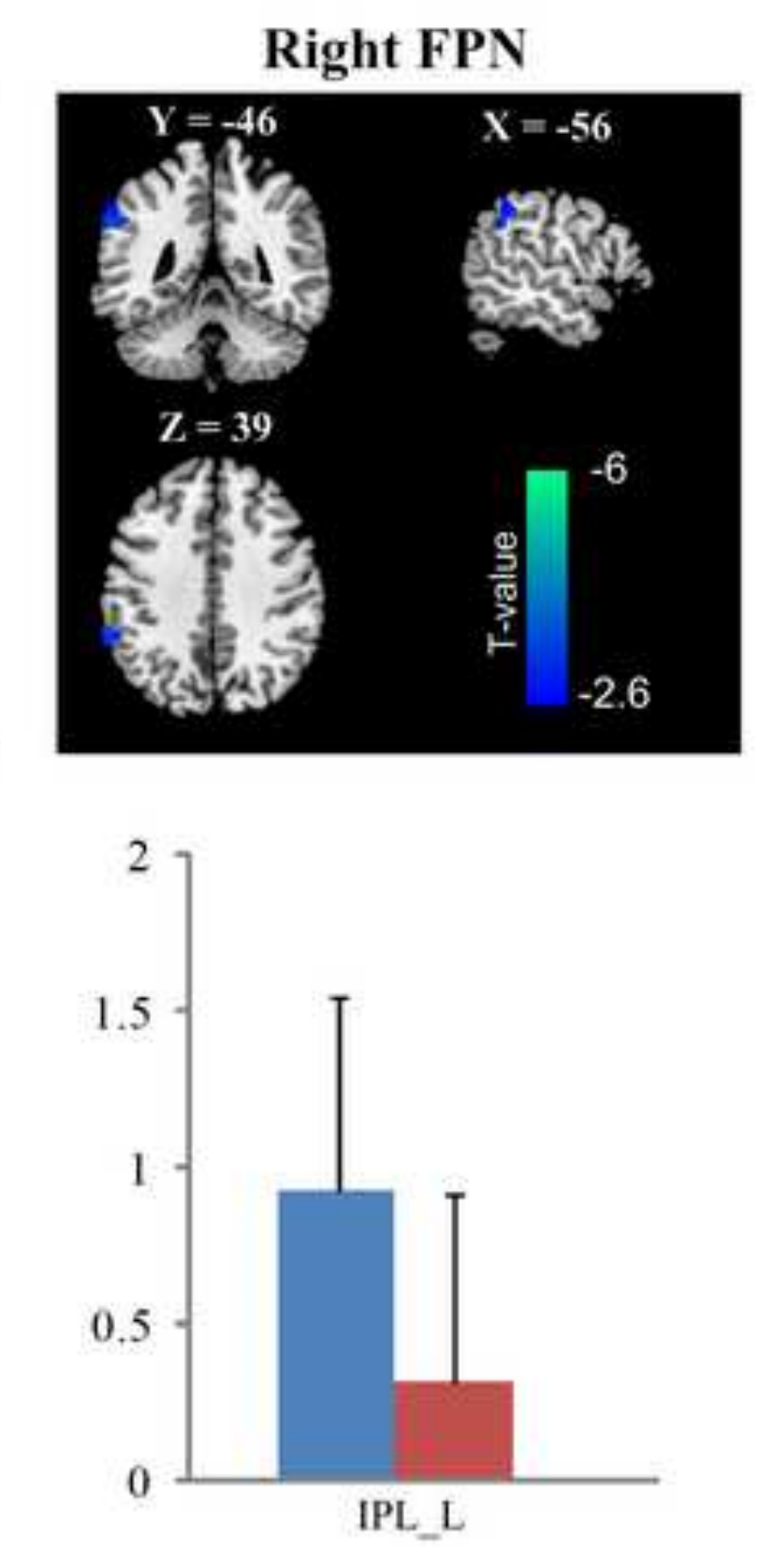

Anterior DMN
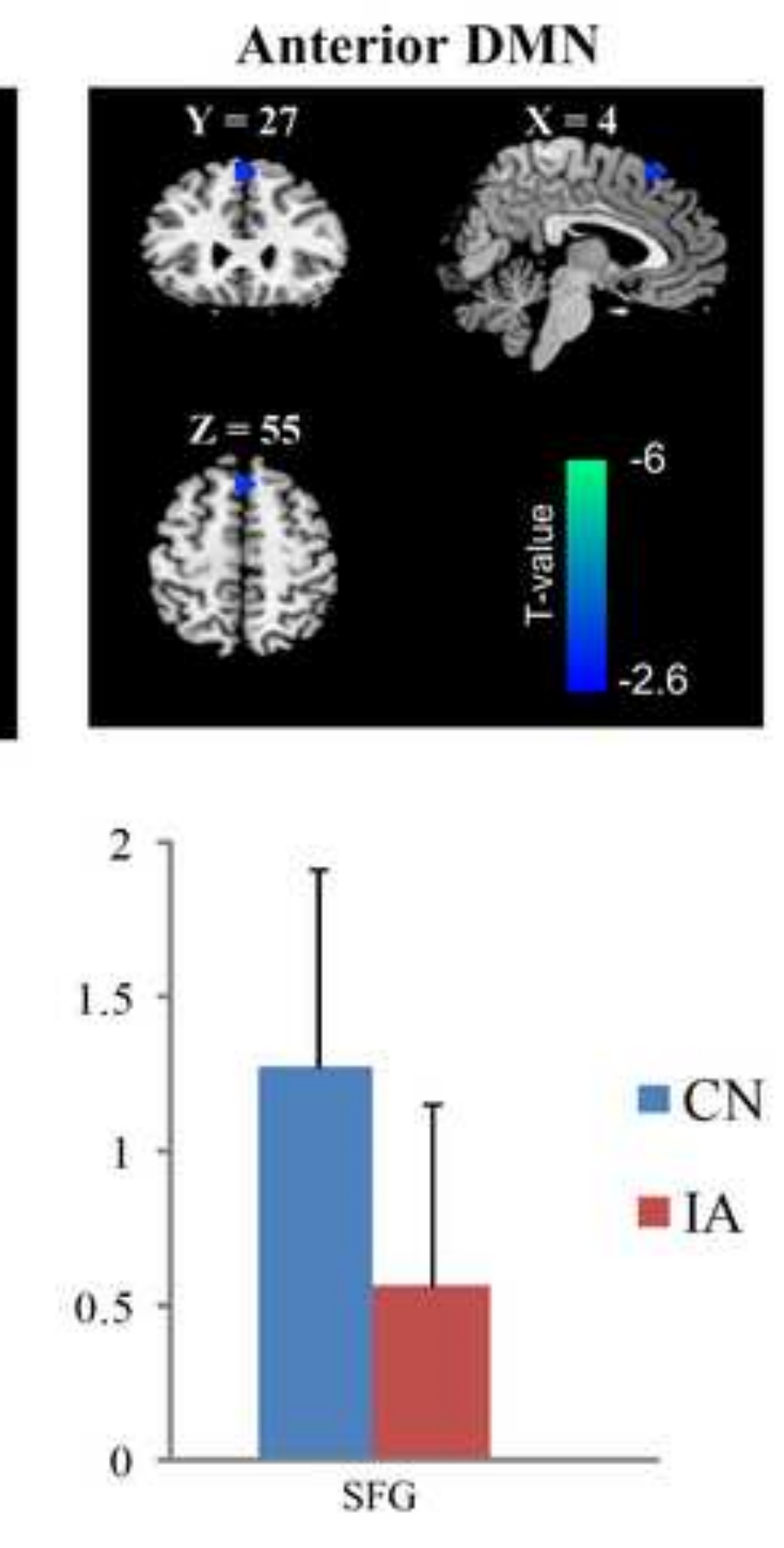

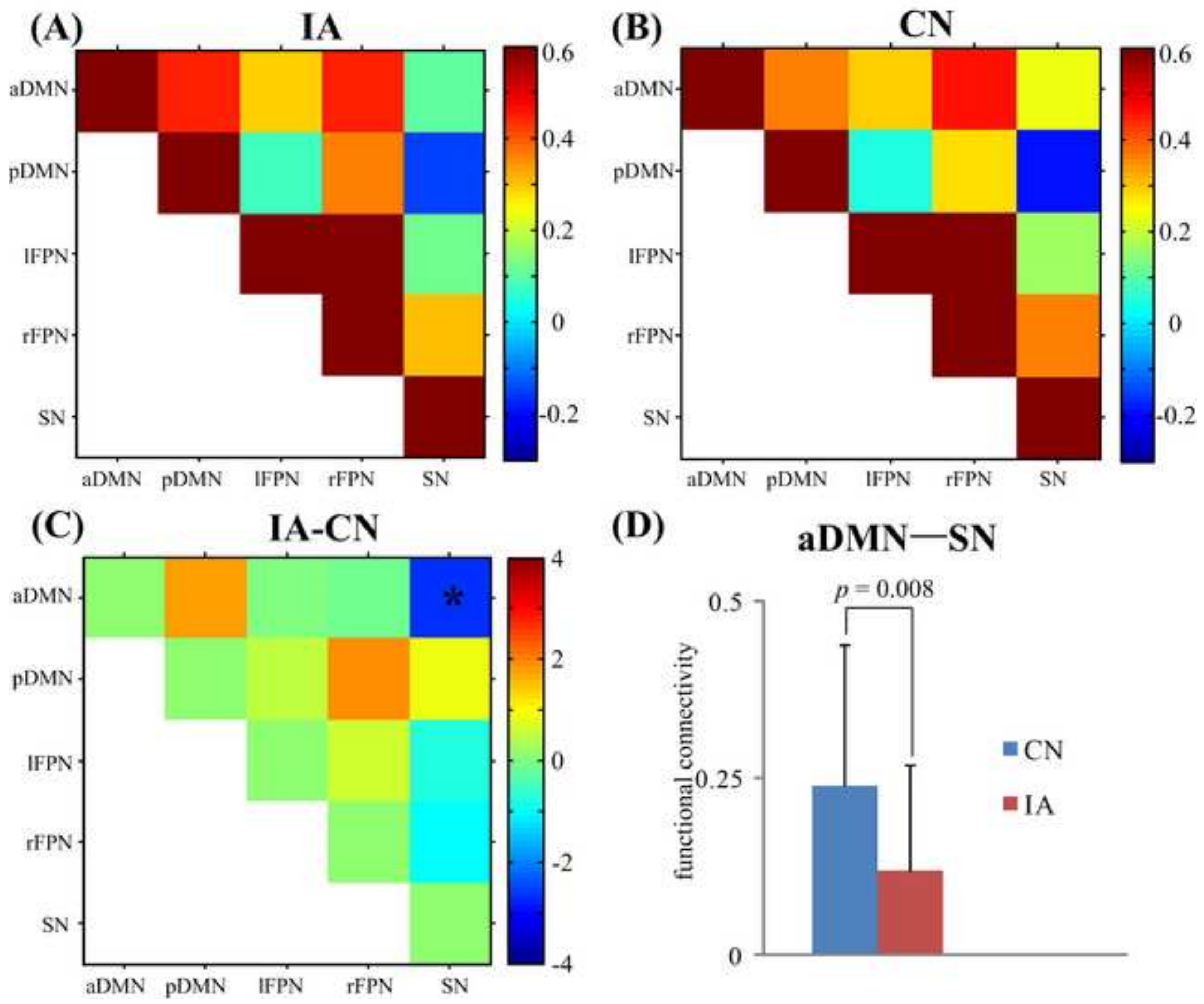

(D)
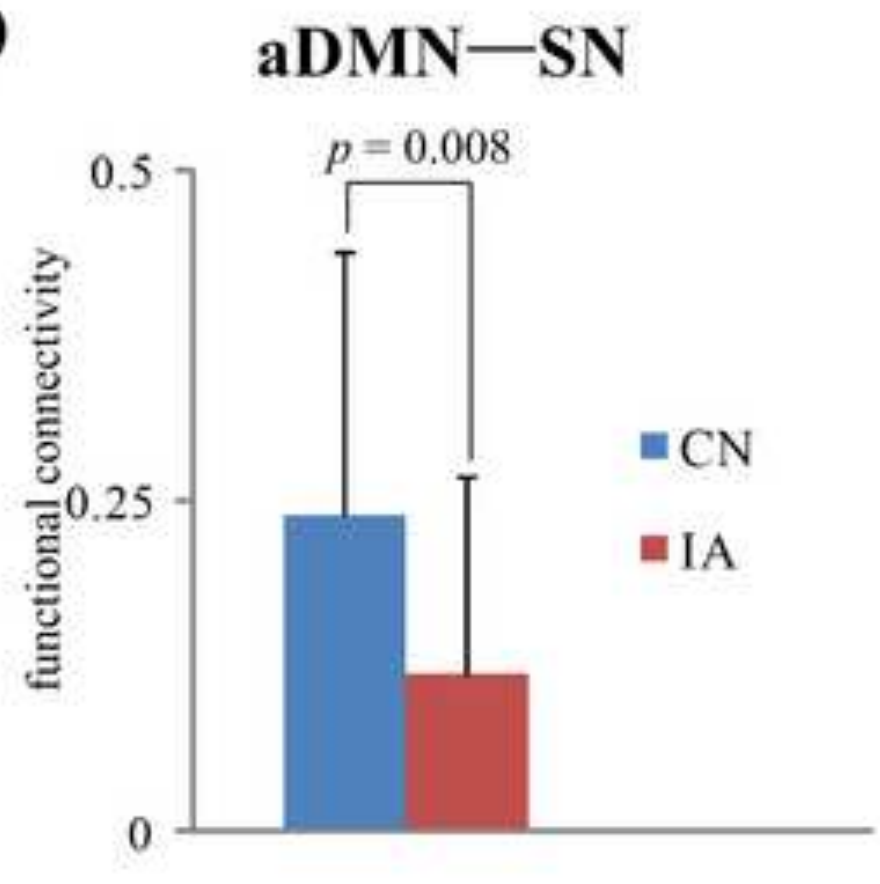